\title{
Real-Time Image Processing Using A Self-Pumped Phase Conjugate Mirror
}

Sze-Keung Kwong, George A. Rakullic, Victor Leyva, Amnon Yariv

Sze-Keung Kwong, George A. Rakullic, Victor Leyva, Amnon Yariv, "RealTime Image Processing Using A Self-Pumped Phase Conjugate Mirror," Proc. SPIE 0613, Nonlinear Optics and Applications, (16 June 1986); doi: $10.1117 / 12.960379$

SPIE. Event: O-E/LASE'86 Symposium, 1986, Los Angeles, CA, United States 
Real-time image processing using a self-pumped phase conjugate mirror

Sze-Keung Kwong, George A. Rakuljic, Victor Leyva and Amnon Yariv

Department of Applied Physics, California Institute of Technology Pasadena, California 91125

\section{Abstract}

Real-time 'exclusive or' operation obtained with an interferometer using a self-pumped phase conjugate mirror is reported. Also, results of image subtraction, intensity inversion and image differentiation are shown. Methods of extending the operation to higher order differentiation and of obtaining the Laplacian are discussed.

\section{Introduction}

In recent years photorefractive crystals have been used to perform various real-time image processing operations. For example, real-time convolution and correlation, ${ }^{1}$ edge enhancement, ${ }^{2}$ image subtraction, ${ }^{3}$ differentiation, ${ }^{4}$ division ${ }^{5}$ and inversion ${ }^{6}$ have been demonstrated. In this paper we report a new method to obtain real-time 'exclusive or' operation and image differentiation based on an interferometer using a phase conjugate reflector.

\section{Image subtraction and 'exclusive or' operation}

Fig. I illustrates the system for 'exclusive or' operation. A wave with amplitude $E_{i n}$ was divided by beam splitter $\mathrm{BS}_{1}$ whose reflection and transmission coefficients are equal to $r$ and $t$ respectively. Without making any assumptions, let $r^{\prime}$ and $t$ ' be the amplitude reflection and transmission coefficients for wave incident from the opposite side of the beamsplitter. Each of the two waves then passed through a transparency with amplitude transmittance $\mathrm{T}_{1}$ for beam 1 and $\mathrm{T}_{2}$ for beam 2. The two beams are then reflected by a selfpumped phase conjugate mirror (SPPCM) with phase conjugate reflectivities $R_{1}$ and $R_{2}$ for beam 1 and beam 2 respectively. ( $R_{1}$ and $R_{2}$ are, in general, not the same.) The phase conjugated beams recombine interferometrically at beam splitter $\mathrm{BS}_{1}$ to form an output field intensity $I_{\text {out }}$ at detector $D_{1}$ given by

$$
I_{\text {out }}=\left.\left|r^{\prime} t^{\star} R_{1}\right| T_{1}\right|^{2}+\left.t r^{\star} R_{2}\left|T_{2}\right|^{2}\right|^{2} I_{\text {in }}
$$

where $I_{\text {in }}=\left|E_{\text {in }}\right|^{2}$

From Stokes' principle of the time reversibility of light

$$
r^{\prime} t^{*}+r^{*} t=0
$$

so that

$$
I_{\text {out }}=\left.\left|R_{1}\right| T_{1}\right|^{2}-\left.R_{2}\left|T_{2}\right|^{2}\right|^{2}\left|r^{*} t\right|^{2} I_{\text {in }}
$$

If the two phase conjugate mirrors are identical, i.e. $R_{1}=R_{2}=R_{\text {, then }}$

$$
\begin{aligned}
I_{\text {out }} & =\left.|| \mathrm{T}_{1}\right|^{2}-\left.\left|\mathrm{T}_{2}\right|^{2}\right|^{2}\left|\mathrm{r}^{*} \mathrm{tR}\right|^{2} \mathrm{I}_{\text {in }} \\
& \propto\left|\mathrm{T}_{1}\right|^{2} \oplus\left|\mathrm{T}_{2}\right|^{2}
\end{aligned}
$$

where $\oplus$ represents the Boolean 'exclusive or' operation. Similarly, the field intensity I' measured by detector $\mathrm{D}_{2}$ is

$$
\left.I^{\prime} \propto|| T_{1}\right|^{2}+\left.|r|^{2}\left(\left|T_{2}\right|^{2}-\left|T_{1}\right|^{2}\right)\right|^{2}|R|^{2} I_{\text {in }}
$$

We note that the $\pi$ phase shift between the complex fields of the two images, which is the key element of the 'exclusive or' operation, is introduced naturally by the time reversibility of light. This is an essential difference between this method and other methods ${ }^{3}$ in 
which the $\pi$ phase shift was artificially provided by a piezo-electric mirror or an electrooptical modulator. This device is only sensitive to intensity differences of the two transparencies or the optical path lengths of the two arms.

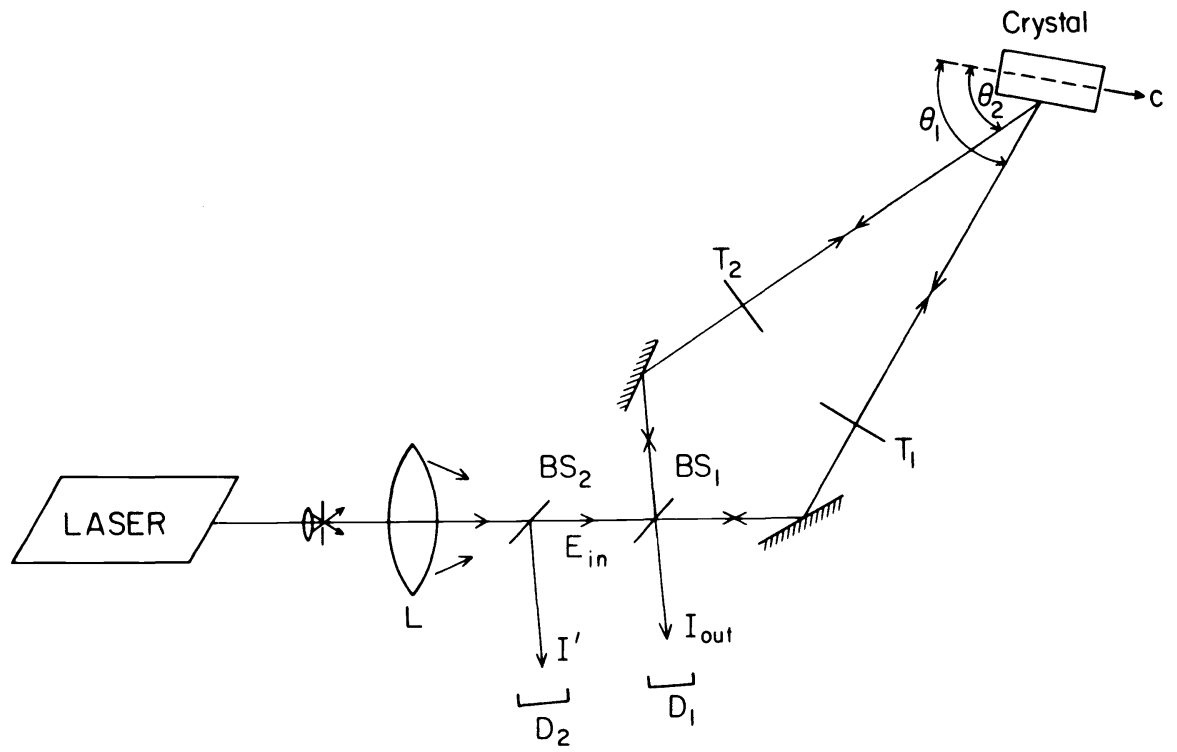

Figure 1. Experimental arrangement to demonstrate the exclusive or operation

In the experiment a single TEMoo mode Argon laser beam (5145A, 50mW) was expanded and split into two beams by $50 \%$ beam splitter $\mathrm{BS}_{1}$. Each beam was then passed through separate transparencies $\mathrm{T}_{1}$ and $\mathrm{T}_{2}$. A lens $\mathrm{L}(\mathrm{F}-30 \mathrm{~cm})$ was used to focus the two expanded beams which were adjusted to overlap completely inside the poled $\mathrm{BaTiO}_{3}$ crystal. The crystal was then aligned to form a self-pumped phase conjugate mirror ${ }^{7}$ by setting the angles between the beams and the crystal C-axis to $\theta_{1}=50^{\circ}$ and $\theta_{2}=40^{\circ}$. The two image bearing beams were phase conjugated simultaneously with no cross talk. ${ }^{8}$ The magnitude of the phase conjugate reflectivities of beam 1 and beam 2 were approximately the same and equal to $25 \%$. The phases of complex phase conjugate reflection coefficients of the two beams are also the same. Since the SPPCM regards the combination of the two input beams as a single complex input wave, and due to the beams' overlap in the crystal they are both reflected from the same set of gratings. Another method for obtaining phase locking between the two phase conjugate beams is described in Ref. 9, in which a self induced oscillation locks the relative phase between the two phase conjugate beams. The phase conjugate reflected image bearing beams were then combined interferometrically at beam splitter $\mathrm{BS}_{1}$. The two transparencies and the detectors were placed close to the beam splitters to reduce diffraction aberration.

The transparencies $\mathrm{T}_{1}$ and $\mathrm{T}_{2}$ used in the experiment are pictures of a semicolon and a colon respectively. The phase conjugate images of these two transparencies are shown in Figures $2 \mathrm{a}$ and $2 \mathrm{~b}$ respectively. Figure $2 \mathrm{c}$ is the image detected by $\mathrm{D}_{1}$, which represents the 'exclusive or' operation (or, in this case, image subtraction) between the two images, $\left|\mathrm{T}_{1}\right|^{2} \oplus\left|\mathrm{T}_{2}\right|^{2}$. Figure $2 \mathrm{~d}$ is the image recorded by $\mathrm{D}_{2}$, which represents $\left.|| \mathrm{T}_{1}\right|^{2}+\left.|\mathrm{r}|^{2}\left(\left|\mathrm{~T}_{2}\right|^{2}-\left|\mathrm{T}_{1}\right|^{2}\right)\right|^{2}$ and is proportional to the sum of intensities, $\left.|| \mathrm{T}_{1}\right|^{2}+\left.\left|\mathrm{T}_{2}\right|^{2}\right|^{2}$, when $|\mathrm{r}|^{2}=.5$. Slight edge enhancement effects ${ }^{2}$ were also observed in these figures which are probably due to large object beam intensities as compared to the weaker pump beam intensities. These results are independent of the optical path lengths of either beam between the BS 1 and the crystal.

The response time of the self-pumped phase conjugate mirror obeyed approximately the relation $\tau \simeq 10 / \mathrm{I}$ shere $I$ is the total intensity of the interaction beams in $\mathrm{mW} / \mathrm{mm}^{2}$. 


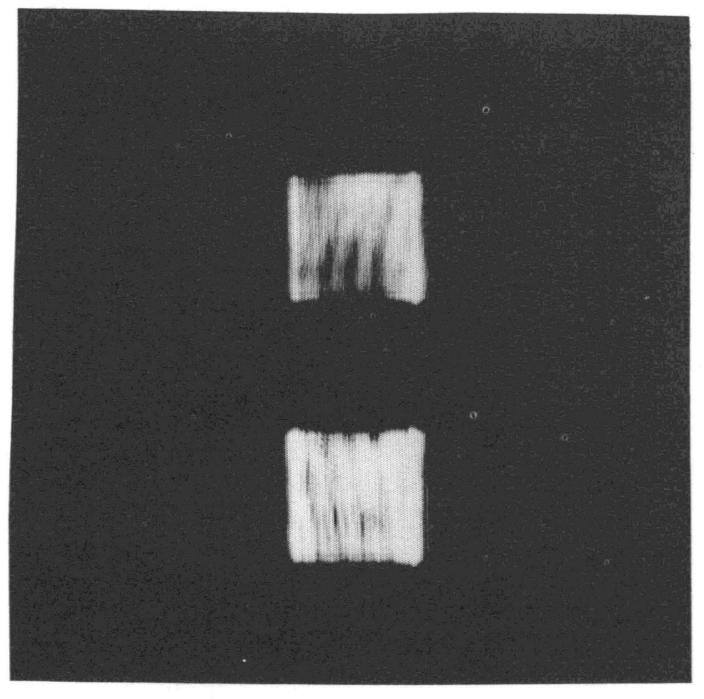

Fig. 2 (a) Image of a semicolon after phase conjugation

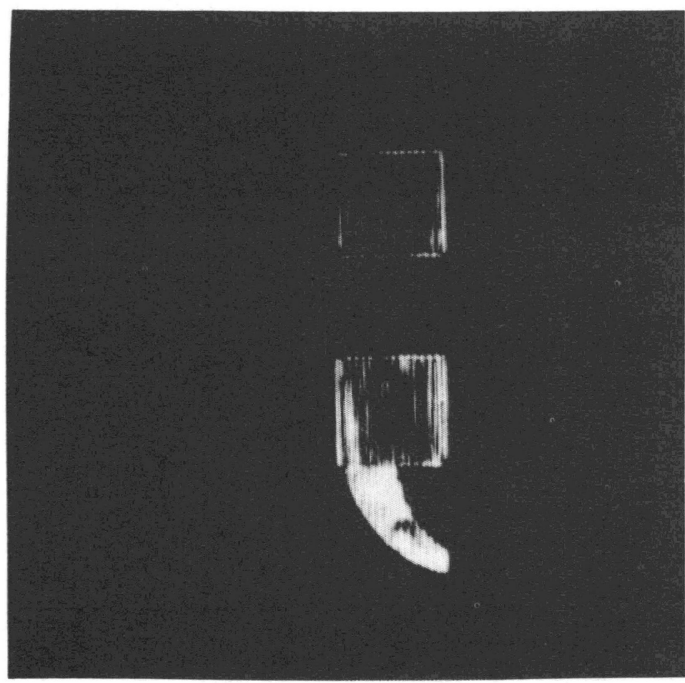

Fig. 2(c) Image subtraction of semicolon and colon

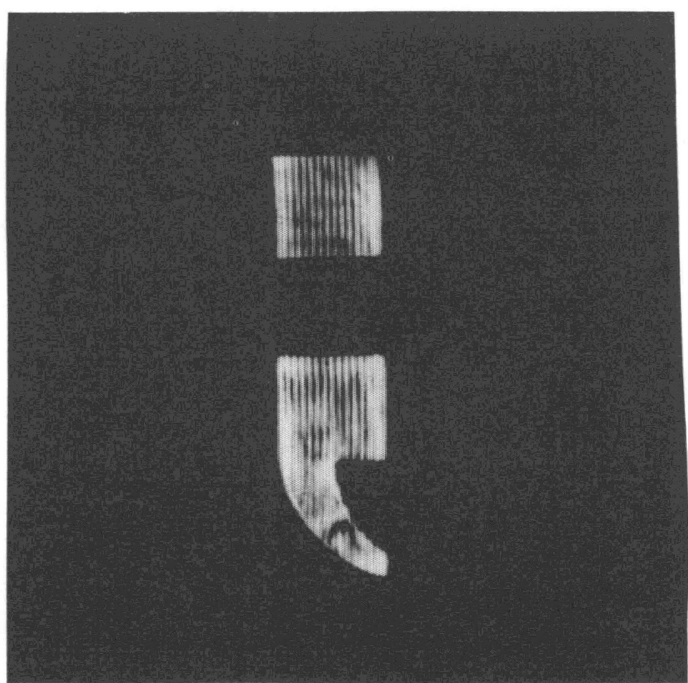

Figure 2(b) Image of a colon after phase conjugation

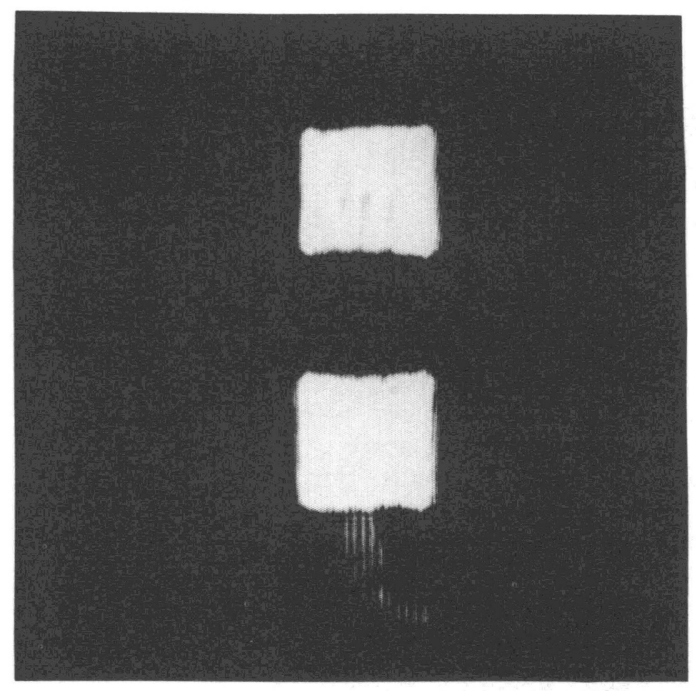

Fig. $2(\mathrm{~d})$ Image detected by detector $\mathrm{D}_{2}$

\section{Intensity inversion}

Optical intensity inversion was also observed by simply removing transparency $\mathrm{T}_{2}$ so that the intensity detected by $D_{1}$ is proportional to $\left.\left.|1-| T_{1}\right|^{2}\right|^{2}$ which result follows from Eq. (3) when we put $\left|\mathrm{T}_{2}\right|^{2}=1$. Figs. $3 \mathrm{a}$ and $3 \mathrm{~b}$ are the phase conjugate images of a uniform illumination and a resolution chart respectively. Fig. $3 \mathrm{c}$ is the intensity inverted image detected by $\mathrm{D}_{1}$. Fig. 3d is the image addition observed by detector $\mathrm{D}_{2}$, which is proportional to $\left.\left.|1+| T_{1}\right|^{2}\right|^{2}$.

Intensity inversion by a different method which uses four wave mixing was reported recently by Ochoa et al.6 In their method the object beam intensity is required to be much larger than that of the reference beam in order to ensure that the diffraction efficiency of the index grating is inversely proportional to the object beam intensity. 


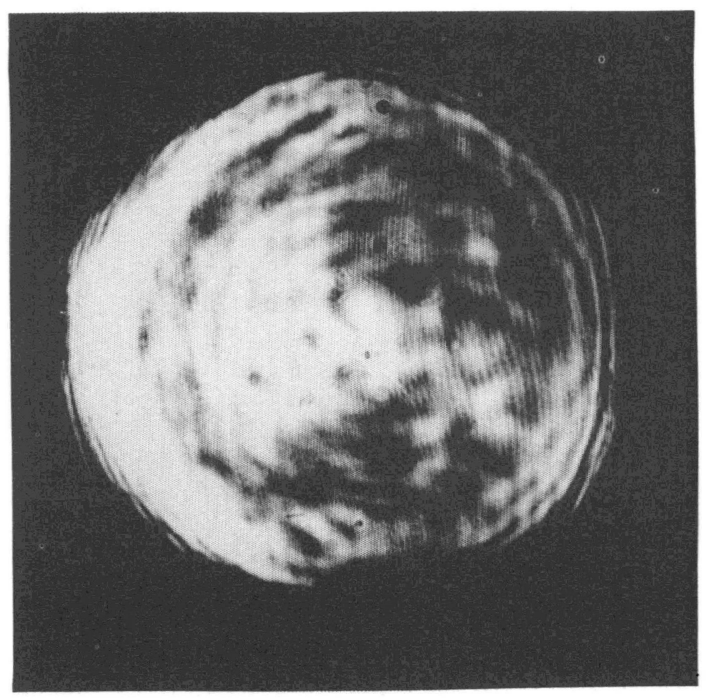

Fig. 3(a) Image of the uniform illumination after phase conjugation

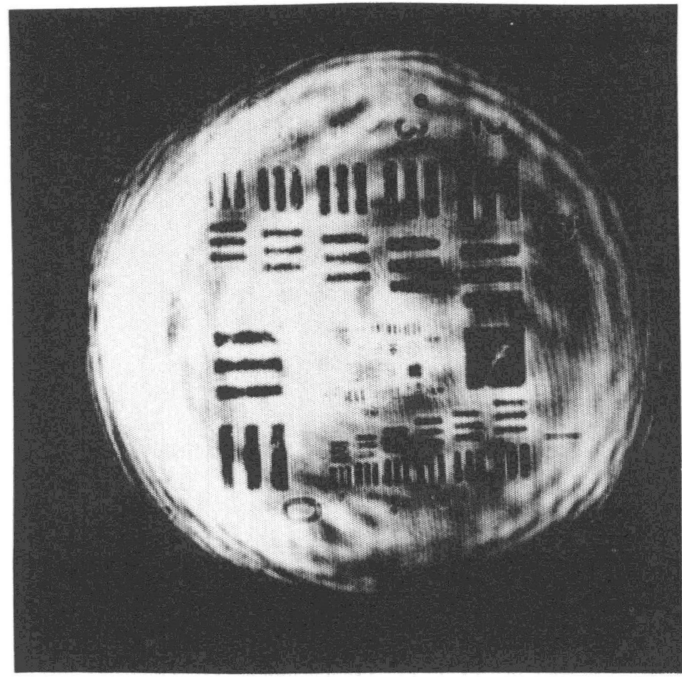

Fig. 3(c) Intensity inversion of the resolution chart

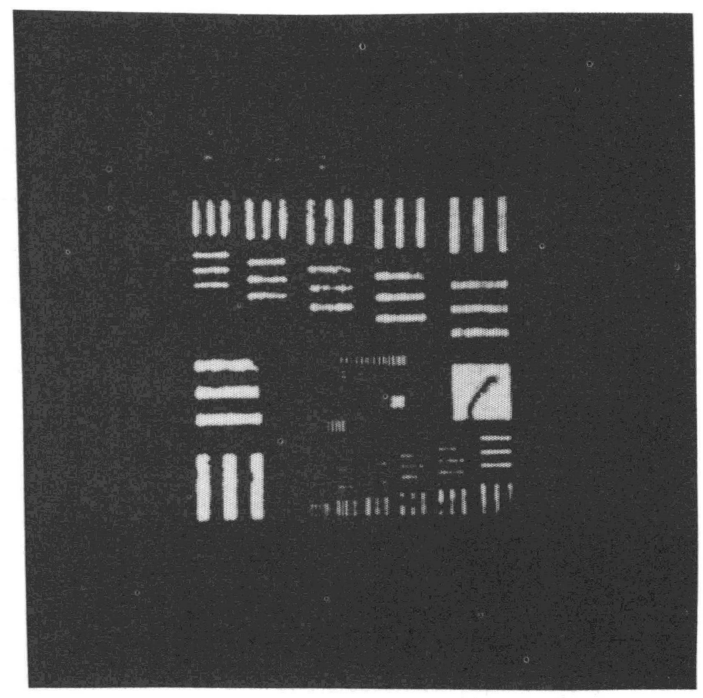

Fig. 3(b) Image of resolution chart after phase conjugation

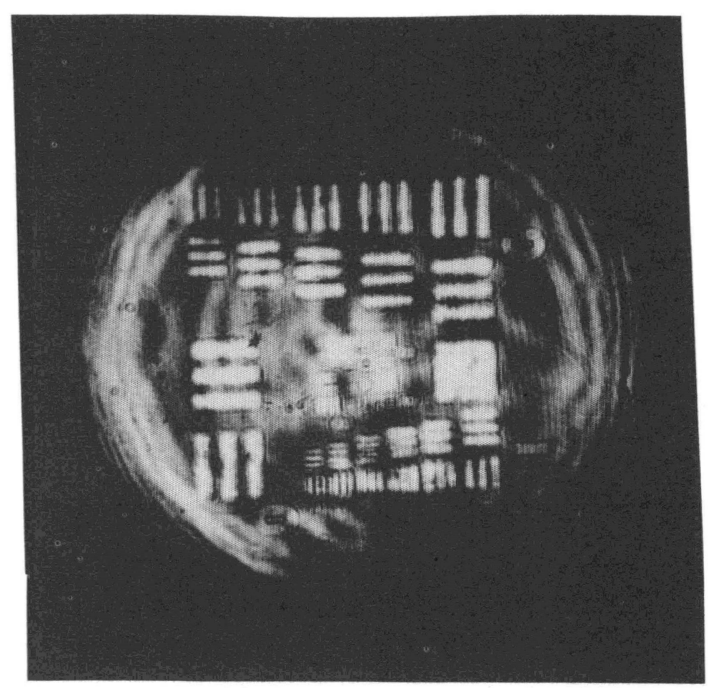

Fig. 3(d) Intensity addition of the resolution chart and the uniform illumination

\section{Image differentiation}

The differential of a function $\mathrm{I}(\mathrm{x}, \mathrm{y})$ can be approximated to any degree of accuracy by using finite differences. Using such a method, the first and second-order differentials are given by

$$
\begin{aligned}
\frac{\partial I(x, y)}{\partial x} & =\frac{I(x+\Delta x, y)-I(x, y)}{\Delta x} \\
\frac{\partial^{2} I(x, y)}{\partial x^{2}} & =\frac{I(x+\Delta x, y)-2 I(x, y)+I(x-\Delta x, y)}{(\Delta x)^{2}}
\end{aligned}
$$

Therefore, the differential of any order can be obtained by adding and subtracting various shifted images of a pattern function. 
The experimental arrangement used to perform first-order differentiation, which is very similar to Figure 1 , is shown in Figure 4. Since the transparency is not placed at the intersection of the two beams, each beam reads a slightly shifted image of the other. The two images are then focused down into the BaTiO 3 crystal which, through total internal reflection, forms a self-pumped phase conjugate mirror. The phase conjugate images are thus phase locked and can recombine at $\mathrm{BS}_{1}$, giving an output amplitude which is proportional to the first order differential. Results are given in Figure 5. Also given in Figure 5 (d) is $(\partial / \partial x+\partial / \partial y)$ obtained by shifting the images by $\Delta x+\Delta y \cdot$

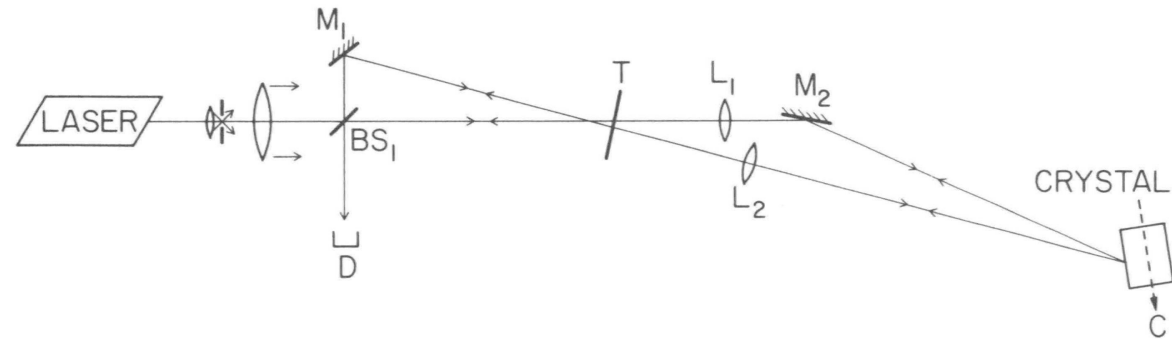

Fig. 4. Experimental arrangement used to demonstrate first order image differentiation

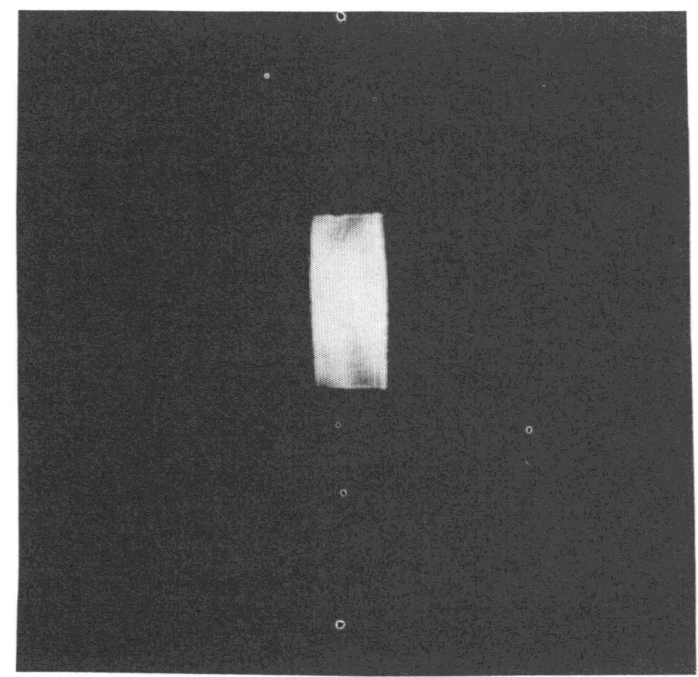

Fig. 5(a) Image of a rectangle after phase conjugation

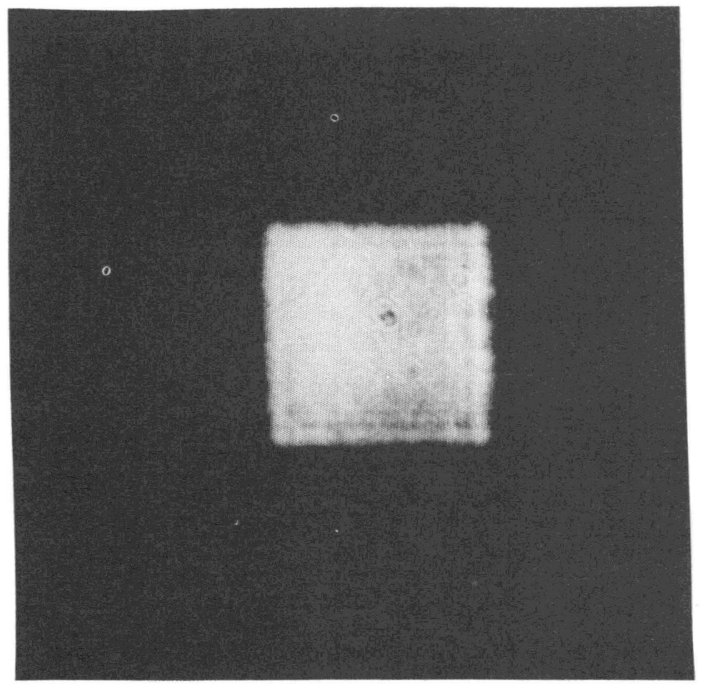

Fig. 5(c) Image of a square after phase conjugation

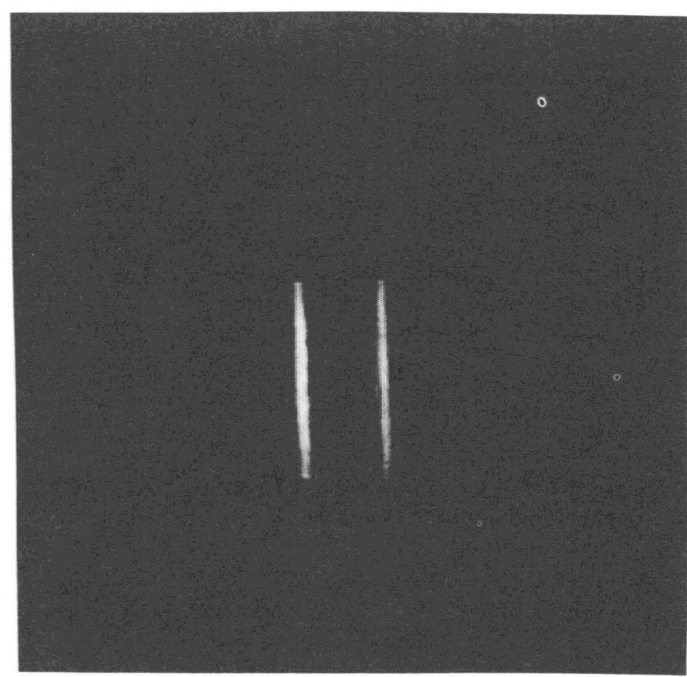

Fig. 5(b) Image of Fig. 5(a) after first-order differentiation

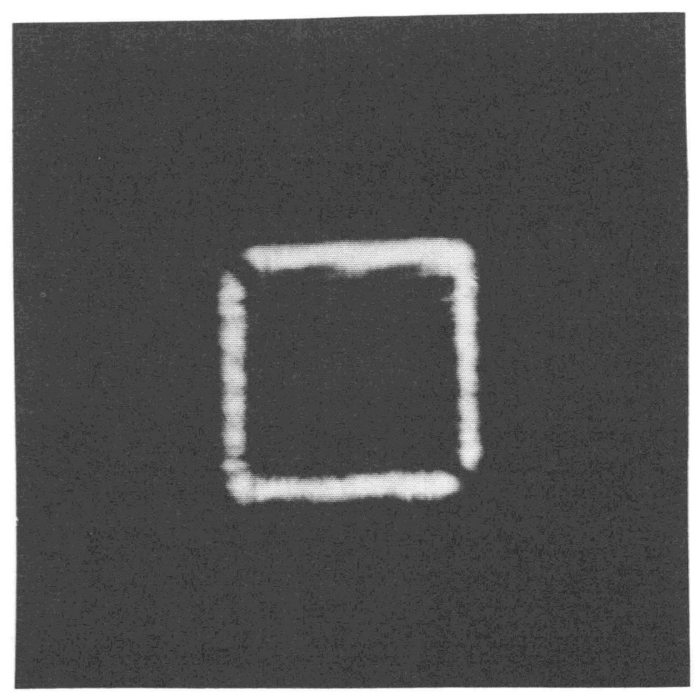

Fig. 5(d) Image of Fig. 5(c) after $\partial / \partial x+\partial / \partial y$ operation

40 / SPIE Vol. 613 Nonlinear Optics and Applications (1986) 
Figure 6 gives the setup used to perform second-order image differentiation. The two outside beams read $T(x+\Delta x, y)$ and $T(x-\Delta x, y)$, while the center beam reads $T(x, y)$. The reflectivity of beam splitter $\mathrm{BS}_{2}$ is $50 \%$, giving an output amplitude at $\mathrm{BS}_{1}$ proportional to the second order differential.

$$
\left.I_{\text {out }} \propto|| \mathrm{T}(\mathrm{x}+\Delta \mathrm{x}, \mathrm{y})\right|^{2}+|\mathrm{T}(\mathrm{x}-\Delta \mathrm{x}, \mathrm{y})|^{2}-\left.2|\mathrm{~T}(\mathrm{x}, \mathrm{y})|^{2}\right|^{2}
$$

Results are given in Figure 7 .

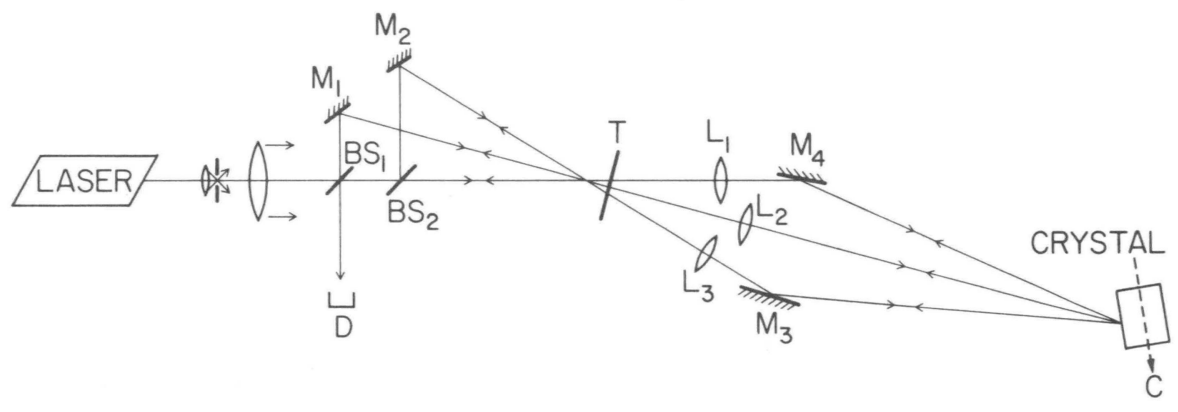

Fig. 6. Experimental arrangement used to demonstrate second order image differentiation

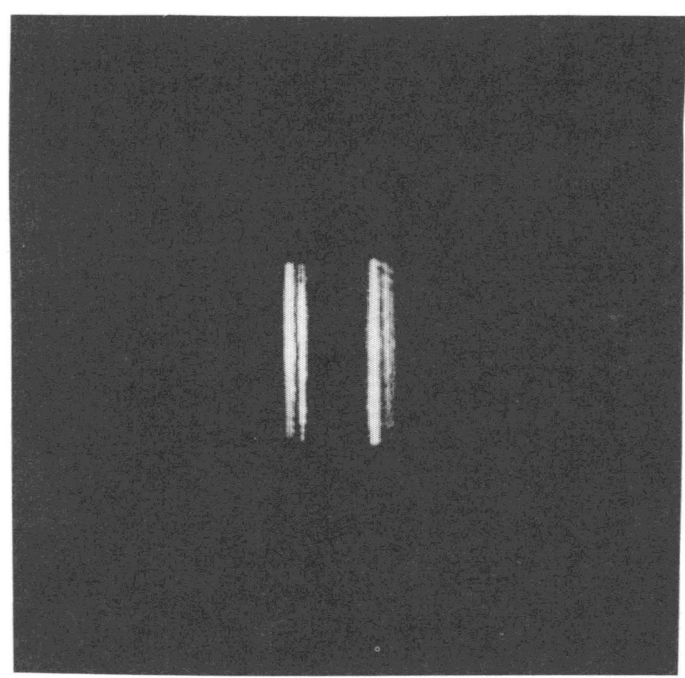

Fig. 7. Image of Fig. 5(a) after second order differentiation

\section{Discussion}

Extensions to higher order image differentiation can be obtained by adding up appropriately shifted images into two groups which are then subtracted at the final beam splitter. While the final beam splitter reflectivity can be arbitrary, the remaining ones must have their reflectivities chosen to perform the image additions in the correct proportions. The many shifted images required for higher order derivatives may cause difficulties in focusing all the images into a single crystal. In that case, two or more crystals phase locked through spontaneous oscillations ${ }^{9}$ could be used to handle the many images. Efforts are also under way to couple the multiple images into the crystal using a single optical fiber.

Using methods similar to those used for image differentiation, a device can be constructed whose output amplitude is proportional to $\nabla^{2} I(x, y)$, where $I=|T(x, y)|^{2}$. A beam is expanded and split into two beams. One of the beams will read $\mathrm{T}(\mathrm{x}, \mathrm{y})$. The other beam will be split into four beams of equal intensity using 50\% beam splitters. These beams will then read images of $\mathrm{T}(\mathrm{x}, \mathrm{y})$ shifted by $\pm \Delta \mathrm{x}$ or $\pm \Delta \mathrm{y}$. These beams will then recombine at the first beam splitter giving an output proportional to $\nabla^{2} \mathrm{I}(\mathrm{x}, \mathrm{y})$. 


\section{Conclusions}

In summary, we have demonstrated a real time 'exclusive or' operation, image subtraction, intensity inversion, and first- and second-order differentiation by an interferometer with a phase conjugate mirror. Methods of extending the operation to higher order differentiation as well as a method for obtaining the Laplacian were discussed.

\section{Acknowledgments}

This work was supported by the U.S. Air Forst office of Scientific Research, the U.S. Army Research Office, Durham, North Carolina and Rockwell International Corporation. V. Leyva would like to thank the support of the Undergraduate Summer Research Fellowship from Caltech.

\section{References}

1. White, J. O., Yariv, A., "Real-time image processing via four-wave mixing in a photorefractive medium," Appl. Phys. Lett., Vol. 37, pp. 5-7. 1980.

2. Feinberg, J., "Real-time edge enhancement using the photorefractive effect," opt. Lett., Vol. 5, pp. 330-332. 1980.

3. Related work by J. P. Huignard, J. P. Herriau and F. Micheron, "Coherent selective erasure of superimposed volume holograms in LiNbo 3 ," Appl. Phys. Lett., Vol. 26, pp. 225$258,1975^{\circ}$ and by $\mathrm{Y}$. H. Ja, "Real-time image subtraction ín four-wave mixing with photorefractive $\mathrm{Bi}_{12} \mathrm{GeO}_{2}$ o crystal," Opt. Comm., vol. 42, pp. 377-380, 1982 employed two sequential exposures of the hologram and was thus not strictly real-time.

4. Ja, Y. H., "Real-time optical image differentiation by degenerate four-wave mixing, Appl. Phys. B, vol. 36, pp. 21-24. 1985 .

5. Ya. Y. H., "Real-time image division in four-wave mixing with photorefractive $\mathrm{Bi}_{12} \mathrm{GeO}_{2}$ o crystal," Opt. Comm., Vol. 44, pp. 24-28. 1982.

6. Ochoa, E., Hesselink, L., Goodman, J. W., "Real-time intensity inversion using two wave and four-wave mixing in photorefractive $\mathrm{Bi}_{12} \mathrm{GeO}_{20}$," Appl. Opt., Vol. 24, pp. 1826-1832. 1985 .

7. In our experiment, a self-pumped phase conjugate mirror using internal reflection at the crystal corner was used (J. Feinberg, "Self-pumped, continuous-wave phase conjugator using internal reflection," Opt. Lett., Vol. 7, pp.486-488, 1982). Other kinds of selfpumped phase conjugate mirror with different geometries (M. Cronin-Golomb, B. Fischer, $\mathrm{J}$. O. White and A. Yariv, "Theory and application of four-wave mixing in photorefractive media," IEEE J. Quant. Electron., Vol. QE-20, pp. 12-30, 1984) also give similar results.

8. Feinberg, J., "Continuous-wave self-pumped phase conjugator with wide field of view," Opt. Lett., Vol. 8, pp. 480-482. 1983.

9. Ewbank, M. D., Yeh, P., Khoshnevisan, M., and Feinberg, J., "Time reversal by an interferometer with coupled phase conjugate reflectors," Opt. Lett., Vol. 10, pp. 282-284. 1985 . 